\title{
An Automatic Approach to Measuring Quality of USB Camera
}

\author{
Jong Yih Kuo, Tai Yu Lai, Chi Han Kao
}

\begin{abstract}
To measure the quality of USB camera needs a lot of time with current operations. Moreover, it might cause some mistakes because of the carelessness from tester. This research proposed an approach to reduce the test time. First, user can set the test chart of customization. Next, the proposed algorithm is applied to identify the test chart and to find the position of pattern. Finally, both the operation actions of tester and measurement time can be reduced.

Keywords--USB camera, automatic measure, autoinspection, intelligence video analysis
\end{abstract}

\section{Introduction}

A lot of products are manufactured quickly by using automatic method. In order to detect the products, each company has its own detection method and process. Company uses the process to training its employees and detecting the products. Traditionally, products are used manual detection. Testers use equipment to detect the product's function and look. The speed of the detection method is slow. The detection result is wrong because human factors. The detection result error will reduce the quality of products and add the manufacturing cost. In recent years, there are automatic and semi-automatic detection method to reducing operation actions of tester and measurement time.

In this study, using intelligent video algorithm to help detection and prevent known problems when the actual manual operation. Intelligent video algorithm is used on manufacturing which needs lots of cost, such as die detection [1] and PCB detection [2]. And the manufacturing model is fixed, such as thread [3] and cutters utilized [4].The above applications are static environment. The static environment is that the environment is changed smaller and can be controlled. The product is detected on automatic detection which is based on image process technology. First, separate the detection area from the non-detecting area. Second, detect the detection area. Finally, output the detection result. The background usually means non-detecting area. And the foreground usually means the detection area. Because the environment can be controlled, such as light, focal distance, noise and the color contrast can be designed in advance. It is convenient the image process technology to separate the detection area. The proposed approach can avoid the manual interference to improve accuracy, because the detection process does not need manual operation.

\section{Literature Review}

With advances in technology, the quality of the products produced by the factory has become increasingly sophisticated and complex, the production speed faster and faster, making the using manual to test the product is a challenge, and manually check the speed is slow. In literature [1], Manual inspection may be because people's fatigue, caused a miscarriage of justice, and the use of manual methods to detect will produce significant personnel costs. In order to reduce the detection error cause by human factors, and reduces the cost of the production. Many researches propose the automatic and semi-automatic detection to solve these problems. Chen [5] proposes a smart machine vision system (SVM). This system finds the feature of PCB by using the design data on CAD. Using the feature to compare with the PCB which needs detection, detect whether the solder attached to the PCB board correctly. Wu [6] and Rau [7] build a PCB board image as a compare template. The template is compared with PCB board to detect the deformation, short circuit, holes and other defects.

For the production of quality products, production equipment detection is very important, in order to prevent reduction in product quality, or damage to the machine, in accordance with the tool life to the timely replacement of cutting tools is very important. Kurada [8] proposes a measurement system. This system adjusts the angle and guide of two fiber endoscope illumination to obtain the comparison between the wear areas and un-wear area to determine the parameters of wear. And then use the image process to find the wear area. The extent of the flank wear land has been determined using both textural and gradient operator. In this study, we applied our previous study [10] to develop the proposed measuring technique.

\section{Measurement Approach}

The purpose of this research is to measure the quality of USB camera. There are sixteen testing items, such as resolution, color accuracy and shading. The functional description is in Table 1. It uses the image that the USB camera screens the testing cards to measure the quality. It separates two steps to measure the quality of USB camera. The detection 
process is in Figure 1.

TABLE 1 TESTING ITEMS

\begin{tabular}{|l|}
\hline (1) Auto exposure \\
\hline (2) Blemish \\
\hline (3) Cluster \\
\hline (4) Color uniformity(=Color shading) \\
\hline (5) Color accuracy \\
\hline (6) Dark corner \\
\hline (7) Dead line \\
\hline (8) Defect pixel \\
\hline (9) Flare \\
\hline (10) Ghost \\
\hline (11) Gray scale \\
\hline (12) Resolution \\
\hline (13) Light axis offset \\
\hline (14) Particle \\
\hline (15) Shading \\
\hline (16) White balance \\
\hline
\end{tabular}



Figure 1. Detection Process

\section{A. Identify Testing Card}

The type of testing cards can be divided into three parts. (1)Testing chart: There are many different patterns in testing chart. The patterns are used to measure the quality of USB camera. (2)Black card: It is a card that the color in card is all block. (3)White card: It is a card that the color in card is all white. The different testing items are used different testing cards. In order to avoid using wrong card to cause detect error, it identifies the testing cards.

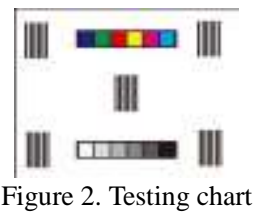

There are sixteen testing items. Eleven testing items use black card and white card to measure quality. Five testing items use testing chart to measure quality. Figure 2 is the testing chat used by this paper. There three patterns in the testing chart, color block, gray color block and MTF pattern. This research proposed an interface to let user to adjust MTF pattern. User can adjust the position and form of MTF pattern and the distance between black and white stripes. The information input by user is to capture the characteristic value of the camera. The process of capture characteristic value is in Figure 3. The characteristic value is used to find the position of MTF pattern. The process of identify testing chart is in Figure 4.



Figure 3. MTF Pattern characteristic

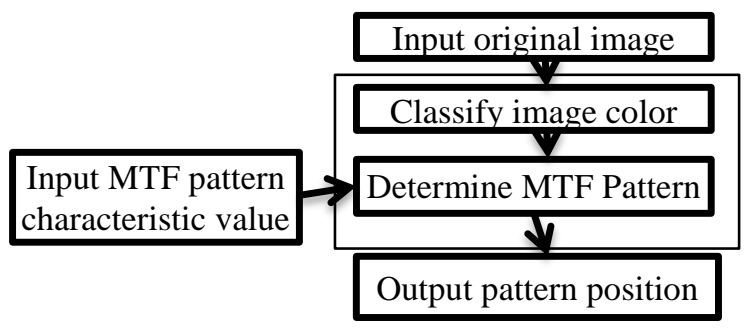

Figure 4. Identify testing chart

\section{The characteristic value of MTF pattern}

User uploads the image when he set the information of MTF pattern. The image is in Figure 5. The system uses the image to analysis the characteristic value. System scans the image from left to right and from top to bottom and calculate the maximum value, minimum value and average value of width of black and white stripes.



\section{Classify Image Color}

This research used Hard C-Means (HCM) to classify the image color. HCM uses the explicit grouping center to classify data. Each data belong to one grouping center. The classify state is 1 or 0 . The initial grouping center may affect the analysis result. But the color of testing chart is formulated by International Commission on Illumination (CIE). The color is fixed, so the initial grouping center can be given according to the classify situation. This research classifies color into two groups. The result separate the black color and non-black color. The algorithm is as follow:

(1) Select grouping center.

(2) Calculate the distance between each data and all grouping center.

(3) Assign data to grouping center. 
(4) Recalculate the grouping center.

(5) Repeat the step from (2) to (4) until grouping center cannot be changed.

The classify result is as Figure 6. The method is used to avoid light effecting the result of measurement.

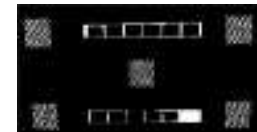

Figure 6. Classify color result

\section{Determine MTF Pattern}

The image that analysis by HCM is scanned from left to right and from top to bottom. The block is marked when it satisfied the characteristic value. Then the neighboring block is linked into a large block by using the morphological processing. In order to find the top left corner and the lower right corner coordinates uses the contour algorithm. Then through the coordinates calculate the black area. The block is removed when the area of block is too large or too small. The remaining blocks are the MTF pattern. The position of MTF pattern is knew. The position of color block and gray color block are fined by using the position of MTF pattern.

Figure 7 is the pseudo code of contour algorithm. $\mathrm{P}$ is the point where we are searching. Direct is the direction of the searching. The searching direction is shown in Figure 8. The start point is the lower left that labeled as 0 . The direction of searching is counterclockwise. If the pixel of searching point is not 255 then search the next direction. If the pixel of searching point then it is the point of block. According to the direction estimate the possible direction of the next point. Using the point and the estimate direction searches the next point. Repeat the steps until the searching and start point are at the same point.

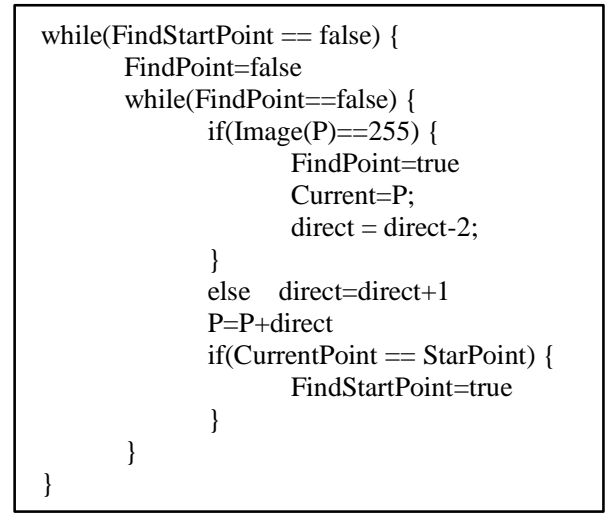

Figure 7. Contour algorithm



Figure 8. Searching direction

\subsection{Black Card and White Card Testing}

The black card and white card are the card with single color. The identify process is shown as Figure 9. First, the image is transformed from RGB color into gray color by using formula (3.1).

$$
\text { Gray }=0.299 \times \mathrm{R}+0.587 \times \mathrm{G}+0.114 \times \mathrm{B}
$$

Then, the color card is identified by using histogram and skewness statistics. The process is shown as follow.



Figure 9. Black card and white card

\section{Reduce the noise}

This study applied the average filter to reduce the noise. The formula is shown as (3.2). AverageFilter(x,y) is the pixel that calculate after average filter. $X$ and $y$ are the position of horizontal and vertical of image. $p(x+i, y+j)$ is the pixel of the image. The values of $i$ and $j$ are between -1 and 1 , because the mask matrix is $3 \times 3$. The average of the nine point that is in the area of mask matrix is calculated as a new pixel to the point.

Figure 10 is the example of average filter. The pixel of red point is lower than the surrounding. It is a noise point. After average filter, the pixel of red point is close to the surrounding. It can reduce the noise.

\begin{tabular}{|c|c|c|c|}
\hline 255 & 189 & 126 & 45 \\
\hline 145 & 45 & 141 & 66 \\
\hline 131 & 112 & 120 & 70 \\
\hline 123 & 68 & 210 & 211 \\
\hline \multicolumn{4}{|c|}{ Before Average Filter } \\
\hline 255 & 189 & 126 & 45 \\
\hline 145 & 140 & 141 & 66 \\
\hline
\end{tabular}




\begin{tabular}{|c|c|c|c|}
\hline 131 & 112 & 120 & 70 \\
\hline 123 & 68 & 210 & 211 \\
\hline
\end{tabular}

After Average Filter

Figure 10. Average filter

$\operatorname{AverageFilter}(\mathrm{x}, \mathrm{y})=\frac{\sum_{i=-1}^{1} \sum_{j=-1}^{1} p(x+i, y+j)}{9}$

\section{Identify Black Card and White Card}

Identifying black card and white card is using histogram to count times that each gray pixel find in the image. The result is analysis by skewness.

Histogram is the method of analysis and statistical that is often used in digital image processing. In this study, the gray image that deals with the average filter is counted the gray value composition by using histogram. Skewness is a measurement value that statistical the distribution of the data and the degree oblique direction. The formula is shown as (3.3). In formula, $\mathrm{n}$ is the total number. It means that is the number of horizontal axis of histogram. If the result is negative then this card is black card. If the result is positive then this card is white card.

$$
\text { Skewness }=\frac{\frac{1}{n} \sum_{i=1}^{n}\left(\mathrm{x}_{\mathrm{i}}-\bar{x}\right)^{3}}{\left(\frac{1}{n} \sum_{i=1}^{n}\left(x_{i}-\bar{x}\right)^{2}\right)^{3 / 2}}
$$

\subsection{Measure Quality of USB Camera Resolution}

First, the RGB image is transformed into gray image. Then, the image is found the edge by using edge detection. The position of MTF pattern is found previously. Then, find the maximum pixel and minimum pixel that is on those positions and on the edge. The result is used formula (3.4) to calculate the result of resolution. Then, the result of resolution is saved to a $\log$ file.

$$
\mathrm{MTF}=\frac{\text { Max pixel }-\min \text { pixel }}{\text { Max pixel }+\min \text { pixel }}
$$

This paper uses sobel filter to do edge detection. Sobel filter uses $3 \times 3$ mask to do edge enhancement. Each pixel and eight neighbor pixel through the mask to calculating the value of edge. The formula is shown as (3.5). In formula (3.5), $\mathrm{P}(\mathrm{x}, \mathrm{y})$ is the point that wants to calculate. SobelFilter $(\mathrm{x}, \mathrm{y})$ is the result of the point $(\mathrm{x}, \mathrm{y})$. After the image deal with sobel filter, the result will deal with the formula (3.6). If the value of SobelFilter(x,y) is bigger than threshold, then the value is 255 . If the value of SobelFilter(x,y) is smaller than threshold, then the value is 0 .

SobelFilter $(\mathrm{x}, \mathrm{y})=$

$$
\begin{aligned}
& \operatorname{Max}\left\{\sum_{i=-1}^{i} \sum_{j=-1}^{i} \mathrm{P}(\mathrm{x}+\mathrm{i}, \mathrm{y}+\mathrm{j}) \times\right. \\
& \left.\operatorname{SobelMask}_{\mathrm{k}}(\mathrm{x}+\mathrm{I}, \mathrm{y}+\mathrm{j}) \mid \forall \mathrm{k}=1 \sim 8\right\} \\
& \text { SobelFilter }(\mathrm{x}, \mathrm{y})=
\end{aligned}
$$

$$
\left\{\begin{array}{r}
0, \text { SobelFilter }(x, y)<\text { Threshold } \\
255, \text { otherwise }
\end{array}\right.
$$

\section{Color Accuracy}

The test chart can be used to measure the Color accuracy. First, the RGB image is transformed into CIE Lab color space. According to the color block position that is found before to measure different between the standard color and the captured color. The different is calculate by formula (3.7).

$$
\Delta \mathrm{E}=\sqrt{\left(\mathrm{L}_{1}-\mathrm{L}_{2}\right)^{2}+\left(\mathrm{a}_{1}-\mathrm{a}_{2}\right)^{2}+\left(\mathrm{b}_{1}-\mathrm{b}_{2}\right)^{2}}
$$

Shading

It uses the white card to detect shading. First, the image is transformed into gray image. The gray image is split into nine blocks. Each block calculates the average of gray pixel. Final, using the formula (3.8) to calculate the rate of each block to center block. Shading is the shading detection value of the $i$ block. GrayAverage $e_{i}$ is gray average value of the $i$ block. The range of number of $i$ is 1 to 9 . The number 5 is the center block.

$$
\text { Shading }_{\mathrm{i}}=\frac{\text { GrayAverage }_{\mathrm{i}}}{\text { GrayAverage }_{5}}
$$

\section{Iv. Experiment Result}

A measurement of the quality of USB camera is developed to verify the effectiveness of our proposed testing method. According to the practice described in chapter III, the identified testing chart can find the position of each pattern. So the tester does not need to select measurement area. This method can reduce the operations for tester and shorten the measurement time.

This paper developed an USB camera measurement system (UCMS) to measure the quality of USB camera. Figure 11 and Figure 12 are the result of location. The type of black and white stripes in Figure 11 is straight. The type of black and white stripes in Figure 12 is oblique. For different type of MTF pattern can be successfully locate. Even if the tester does not put the testing chart in the middle of the screen, it can still be successful on the identification and location testing chart. The result is shown as Figure 13.

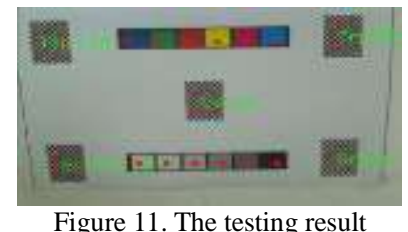

Figure 11. The testing result 


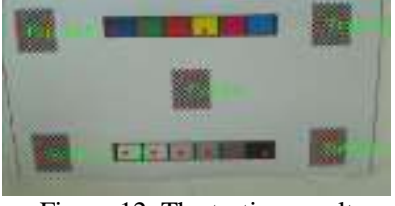

Figure 12. The testing result

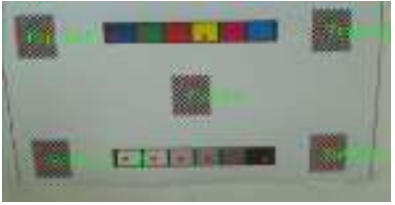

Figure 13. The testing result

\section{v. Conclusion}

To measure the quality of USB camera needs a lot of time with current operations. Moreover, it might cause some mistakes because of the carelessness from tester. This study developed a USB camera measurement system to measure the quality of USB camera, so that tester may reduce the test time. First, user can set the test chart of customization. Next, the proposed algorithm is applied to identify the test chart and to find the position of pattern. Finally, both the operation actions of tester and measurement time can be reduced.

\section{Acknowledgment}

This work was supported by the Ministry of Science and Technology, under grant number 103-2221-E-027-064.

\section{Reference}

[1] C.Y. Chang, C.H.Li, J.W. Chang, and M.D. Jeng "An unsupervised neural network approach for automatic semiconductor wafer defect inspection," Expert Systems with Applications, vol. 36, Issue 1, pp. 950-985, 2009.

[2] X.X. Gao, C.Z. Zhao, G.R. Chang, and Z.H. Tan, "Design and Implementation of Drill Hole of PCB Detection System," Ninth International Conference on Hybrid Intelligent Systems, pp. 462-466, 2009.

[3] D.B. Perng, S.H.Chen, and Y.S. Chang, "A novel internal thread defect auto-inspection system," The International Journal of Advanced Manufacturing Technology, vol. 47, Issue 5-8, pp. 731-743, 2010.

[4] J. C. Su, C. K. Huang, and Y. S. Tamg, "An automated flank wear measurement of microdrills using machine vision," Journal of Materials Processing Technology, vol. 180, Issue 1-3, pp. 328-335, 2006

[5] T.Q. Chen, J.Zhang, Y.Zhou, and Y.L. Murphy, "A Smart Machine Vision System for PCB Inspection," Engineering of Intelligent Systems Lecture Notes in Computer Science, Vol. 2070, pp. 513-518, 2001.

[6] W.Y. Wu, M.J. J. Wang, Y.Zhou, and C.M. Liu, "Automated inspection of printed circuit boards through machine vision," Computers in Industry, vol. 28, pp. 103-111, 1996.

[7] H.Rau, and C.H. Wu, "Automatic optical inspection for detecting defects on printed circuit board inner layers," The International Journal of Advanced Manufacturing Technology, vol. 25, Issue 9-10, pp. 940-946, 2005.
[8] S.Kurada, and C.Bradley, "A machine vision system for tool wear assessment, Tribology International," Tribology International, vol. 30, Issue 4, pp. 295-304, 1997.

[9] S. Chen, B. Lin, X. Han, and X. Liang, "Lip Detection and Tracking Using Variance Based Haar-Like Features and Kalman filter," 2010 Fifth International Conference on Frontier of Computer Science and Technology, pp. 608-612, 2010.

[10] Jong Yih Kuo, Tai-Yu Lai, Yong-Yi Fanjiang, Fu- Chu Huang, Yi- Han Liao, A Behavior-Based Flame Detection Method for a Real-Time Video Surveillance System, Journal of the Chinese Institute of Engineers, 2015.

Jong Yih Kuo, Tai Yu Lai, Chi Han Kao, Department of Computer Science and Information Engineering, National Taipei University of Technology, Taipei, Taiwan, email: jykuo@ntut.edu.tw. 